\title{
Gastric leiomyosarcoma: Report of a case
}

ROBERT D. MCGARRIGLE, D.O

EDWARD A. GOTFRIED, D.o., FACOS

Springfield, Pennsylvania

The symptoms of weakness, fatigue, epigastric pain, and weight loss are nonspecific. The demonstration of a lobulated, submucosal gastric tumor on upper gastrointestinal tract x-ray should prompt the consideration of a gastric leiomyosarcoma.

Leiomyosarcomas of the stomach are infrequently encountered tumors. They represent 1 to 3 percent of all gastric neoplasms, and, despite advances in endoscopy and computerized tomography, early diagnosis is accomplished rarely. ${ }^{1}$ The tumor often remains clinically silent until it achieves remarkable size, by which time the bulk of the neoplasm is extragastric.

This paper presents a case report and a discussion of the presenting complaints, thorough diagnostic workup, and surgical management of gastric leiomyosarcomas.

\section{Report of a case}

A 47-year-old black woman presented with chief complaints of weakness, dizziness, lethargy, and menorrhagia. Five days prior to admission, the patient had developed heavy vaginal bleeding, which had lasted approximately 3 days. She had been amenorrheic for approximately 6 months; prior to then, her menses were regular and without heavy bleeding. She did admit to some fatigue over the previous 4 to 5 months, but she attributed this to overworking. She denied any history of abdominal pain, melena, or rectal bleeding. She did admit to a weight loss of approximately 10 pounds over 2 months' duration, but she stated that she had been on a diet.

The patient's past medical history was significant for sinusitis and a urinary tract infection, which had required hospitalization. She denied any previous surgery. She was gravida I, para 1-0-0-1. She had no known drug allergies, and medications consisted of vitamins only. She had stopped smoking and drank alcohol socially. The patient's family history was remarkable in that her father had died of leukemia in his 50's, while her mother had died in her 70's from an unknown carcinoma of the gastrointestinal tract.

\section{Physical examination}

The patient was $5 ' 5$ " tall and weighed 185 pounds. She appeared well nourished and was in no acute distress. Her vital signs were as follows: blood pressure, 110/68 $\mathrm{mm}$. $\mathrm{Hg}$; pulse rate, 102 beats/minute; respiratory rate, 20 /minute; and temperature, 99.1 F. The HEENT examination revealed her conjunctiva to be pale. Her neck and breasts were without masses. Her heart was regular, with a grade $2 / 6$ systolic ejection murmur. Her lungs were clear in all fields. The abdomen was soft and obese, and bowel sounds were present. No hernias or masses were palpated. Vaginal examination revealed an enlarged, retroverted uterus with nonpalpable adnexa. The extremities demonstrated no clubbing, cyanosis, or edema, and the neurologic examination revealed no focal deficits. Structural examination revealed paraspinal musculature spasm at L1 through L5.

The patient's hemoglobin concentration/hematocrit reading were $4.3 \mathrm{gm} . / \mathrm{dl}$. and 14.5 percent, respectively. The reticulocyte count was within normal limits, and indices were consistent with hypochromic, microcytic iron deficiency anemia. Admission serum electrolyte values were normal except for a potassium level of 3.3 $\mathrm{mEq} . / \mathrm{L}$.(normal, 3.5-5.5 mEq./L.). The metabolic profile revealed a serum albumin level of $3.2 \mathrm{gm} . / \mathrm{dl}$. (normal, 3.4-4.4 gm./dl.) and a total serum protein value of $5.8 \mathrm{gm}$./ dl. (normal, 5.9-8.1 gm./dl.). Serum folate and vitamin $B_{12}$ levels were within normal limits.

The chest $\mathrm{x}$-ray was normal, and the electrocardiogram revealed sinus tachycardia. The serum iron level was 15 $\mathrm{mcg}$./dl. (normal 65-156 mcg./dl.), and the total iron binding capacity was $288 \mathrm{mcg} / \mathrm{dl}$. (normal, $250-410 \mathrm{mcg}$./dl.), with a saturation of 5 percent.

\section{Hospital course}

The patient was admitted with a diagnosis of anemia, and consultation from the gynecology and hematology services was obtained. The gynecologic evaluation suggested that the vaginal bleeding was secondary to dysfunctional uterine bleeding and uterine myoma, but that the severe anemia could not be attributed to these alone. Recommendations included ultrasonography, intravenous urography, barium enema, and dilation and curettage.

The patient was started on intravenous fluids and oral iron supplements and was transfused with 4 units of packed red blood cells during her first 2 hospital days, with the hemoglobin concentration rising to $9.7 \mathrm{gm} . / \mathrm{dl}$. She remained afebrile, and her vital signs were stable. Ultrasonography of the pelvis confirmed the presence of a large, fibroid uterus. On the fourth hospital day, barium enema and intravenous pyelography revealed a large pelvic mass compressing both ureters and sigmoid colon. 
Gastroenterology consultation was ordered, and a diagnosis of anemia secondary to chronic gastrointestinal blood loss was made. Upper endoscopy was performed, and a $3 \times 2 \mathrm{~cm}$. mass was identified along the greater curvature of the body of the stomach, approximately 10 $\mathrm{cm}$. below the esophagogastric junction. There was superficial ulceration at the tip of the mass, with fresh blood present. Several biopsy specimens of the lesion were obtained, and a pathologic diagnosis of acute and chronic inflammation of the lamina propria was made. The endoscopist's impression was that the lesion was benign and felt soft on biopsy. Surgical consultation was ordered on the fifth hospital day.

The patient's hemoglobin concentration at this time was stable, at $10.1 \mathrm{gm}$./dl. She was transfused with an additional 2 units of packed red blood cells, to a level of $12.5 \mathrm{gm} . / \mathrm{dl}$. Upper gastrointestinal roentgenography identified two intramural masses on the greater curvature of the body of the stomach (Fig. 1). The larger, more proximal lesion was noted to have an ulceration within it. On the seventh hospital day, the patient underwent dilation and curettage and cervical biopsy to rule out cervical or endometrial carcinoma as the cause of her profound anemia. Uterine scrapings and cervical biopsy demonstrated proliferative endometrium and mild, chronic inflammation, with no evidence of malignancy.

On the eleventh hospital day, with a preoperative diagnosis of gastric neoplasm, the patient underwent abdominal exploration. An upper midline incision was made, and, upon opening the abdominal cavity, a tumor mass measuring approximately $16 \mathrm{~cm}$. in diameter was seen to protrude from the greater curvature of the stomach. The tumor mass appeared lobulated and purplish-grey in color (Figs. 2 and 3). Necrotic debris and blood clot were present within the tumor mass. There was no evidence of liver metastasis. A large uterine fibroid was identified. The tumor was dissected free from surrounding tissue; there was no invasion of tumor in the lesser omental sac. Radical subtotal gastrectomy and antecolic gastrojejunostomy were performed. The resected margins of stomach and lymph nodes were negative for tumor invasion.

\section{Postoperative follow up}

The patient's postsurgical course was unremarkable, and she was discharged on the eighth postoperative day. Follow-up computed tomographic scanning of the abdomen and pelvis was performed approximately 2 months after discharge and revealed no evidence of metastatic disease. Large uterine fibroids were seen on the pelvic scan.

Eight months after gastric surgery, the patient was readmitted for total abdominal hysterectomy with bilateral salpingo-oophorectomy for persistent menometrorrhagia and pelvic pain. The pathologic diagnosis revealed multiple intramural leiomyomata uteri, chronic cervicitis, and normal ovaries and tubes. During surgery, the abdomen was explored, and no evidence of metastatic disease was palpable. The patient continues to be followed clinically and with computed tomography yearly to ascertain any evidence of metastatic disease.

The M.D. Anderson data revealed that 76 percent of the

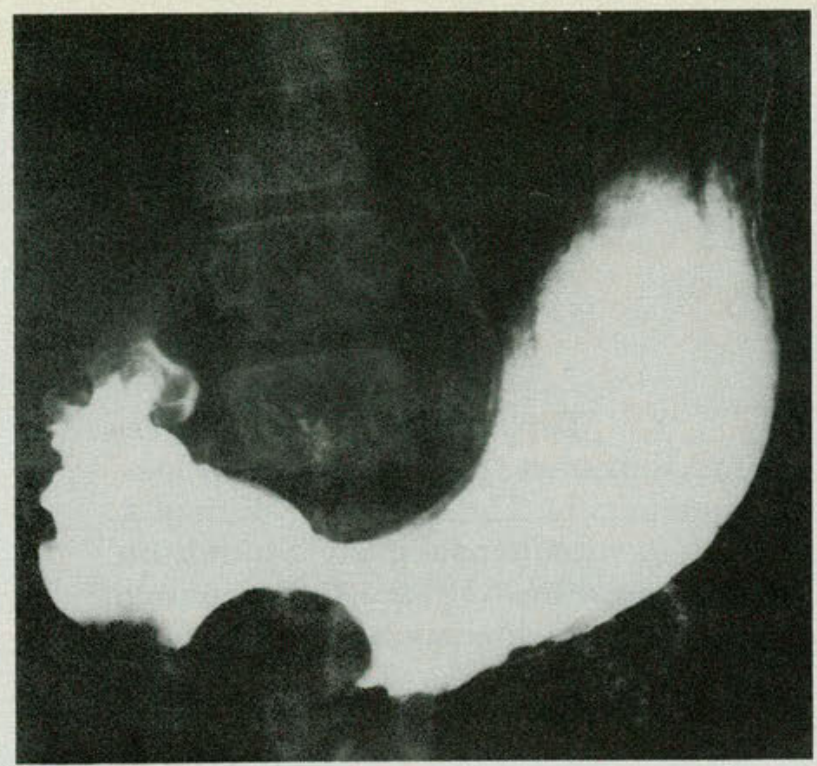

Fig. 1. Lobulated submucosal gastric leiomyosarcoma as demonstrated on upper gastrointestinal tract study.

patients developed metastasis after being followed for at least 4 years. The overall 5-year survival rate for gastric leiomyosarcoma is not much better than for a gastric carcinoma. $^{2}$

\section{Discussion}

Gastric leiomyosarcoma, an unusual neoplasm of smooth muscle origin, accounts for 1 to 3 percent of gastric malignancies. ${ }^{1}$ The median age for occurence of this neoplasm is 54 years; there is a 2 to- 1 male predominance and no hereditary tendency for this neoplasm. ${ }^{1}$

The most frequent symptoms associated with gastric leiomyosarcoma are weakness, gastrointestinal bleeding, pain, epigastric distress, and weight loss. The duration of symptoms before patients seek treatment averages approximately 6 months. ${ }^{2}$ Abdominal mass and/or tenderness are the most frequent positive physical findings. Often, no abnormalities are found on physical examination.

Diagnostic radiographic studies used to confirm the presence of gastric leiomyosarcoma include upper celiac gastrointestinal.roentgenography and endoscopy, selective celiac arteriography, and, most recently, computerized tomography. Many cases of gastric leiomyosarcoma have the same radiographic appearance as benign leiomyomas, and diagnosis must be made histologically. These mural lesions have a smooth, sharply marginated surface, which is usually quite distinct from the irregular, nodular surface of other gastric carcinomas. ${ }^{3}$ Growth of the lesion away from the 

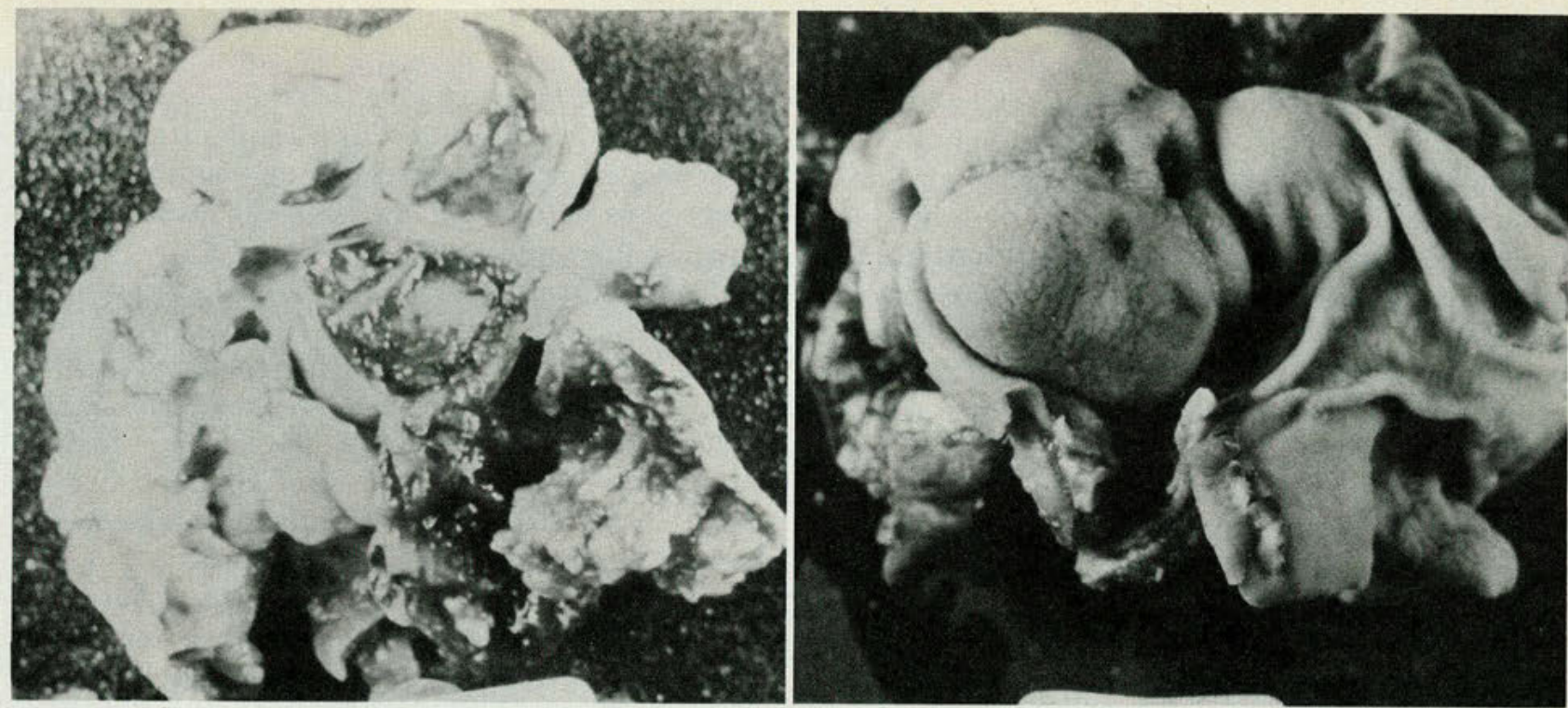

Fig. 2. Gastric leiomyosarcoma extending from greater curvature of stomach into omentum. Fig. 3. Interior of stomach demonstrating submucosal lobulated leiomyosarcoma.

lumen may demonstrate a prominent, exogastric mass that displaces adjacent structures away from the stomach. Ulcerations in the tumor may occur. Biopsy of the lesion at endoscopy has a low diagnostic yield ( 40 percent). ${ }^{2}$ The superficial nature of the bite obtained with conventional endoscopic biopsy forceps is a major limiting factor in diagnosis. The consistent computed tomographic findings of gastric leiomyosarcoma are a large extragastric mass with central necrosis, direct tumor extension into adjacent organs, mesenteric and omental spread, and liver metastasis. ${ }^{4}$ Another feature of this malignancy on computed tomography is the presence of gas bubbles or air fluid levels within the exogastric mass. Also, there is an absence of perigastric lymphadenopathy, which is present in adenocarcinoma and lymphoma of the stomach.

In a review by Lindsay and associates ${ }^{2}$ of 50 gastric leiomyosarcoma patients at M.D. Anderson Hospital and Tumor Institute in Houston, 33 resected specimens were reported to range in size from 2 to $35 \mathrm{~cm}$. in diameter. Fifteen of these were more than $15 \mathrm{~cm}$. in diameter, thus demonstrating their growth to a large size before discovery.

These neoplasms may arise from any part of the stomach, with the majority being well circumscribed and lobulated. No true capsule exists in these tumors. Anterior wall and greater curvature lesions tend to invade omentum and transverse colon, while lesser curvature and posterior wall lesions extend into pancreas, retroperitoneal tissues, and liver. These neoplasms involve the muscularis propria and submucosa and may appear as an endogastric mass. Frequently, the mucosa overlying the tumor will be ulcerated. Central necrosis and hemorrhage are common; these often produce large cysts filled with blood and debris.

The reported rate of metastasis in gastric leiomyosarcoma has ranged from 20 to 100 percent. $^{2}$ The liver, omentum, pancreas, and colon are the most common sites of spread by direct invasion. Lindsay and associates ${ }^{2}$ found no lymph node metastasis in their 50 cases, including patients in whom the tumor was large or involved contiguous organs. This suggests a hematogenous route of spread. These authors also concluded that tumor size was not significant in predicting survival; however, there was a trend toward longer survival with smaller sized neoplasms. Mean survival time was 40 months when the neoplasm was $\leqslant 10 \mathrm{~cm}$. in diameter, and 22 months when the tumor was $>10$ $\mathrm{cm}$. in diameter. Survival time was found to be longer if cellular atypia was mild rather than moderate or severe, or if the number of mitoses was few or moderate. Their data showed no significant survival difference among tumors of epithelioid, spindle, or pleomorphic cell types. Ranchod and Kempson, ${ }^{5}$ in their review of 100 cases, found the frequency of mitoses to be the most useful single criterion for the diagnosis of gastric leiomyosarcoma ( 5 or more mitoses/10 high power field). All tumors with 5 or more mitoses/ 10 high power field behaved in an aggressive manner. 
Surgical removal remains the treatment of choice for gastric leiomyosarcoma. Appleman and Helwig ${ }^{6}$ found that the extent of surgical resection had no effect on survival, and their recommended therapy is to resect as little as possible while removing the entire tumor. Because of the rarity of nodal metastasis with gastric leiomyosarcoma, radical node dissection is not necessary to remove all gross or microscopic disease. Lindsay and associates $^{2}$ obtained the best 5 -year survival rate -43 percent in those patients undergoing distal subtotal gastrectomy. Their overall 5-year survival rate was 19 percent. This study also showed the average times for first recurrence to be 27 months for local reappearance and 16 months for metastasis.

Adjuvant therapy of this tumor has not been in use long enough or often enough to assess its value. While chemotherapy can be effective in reducing the incidences of distant metastases for leiomyosarcomas of other sites, its role in the therapy of gastric leiomyosarcoma is unclear. Johnson and coworkers ${ }^{7}$ reported a complete regression of liver metastasis in a patient with gastric leiomyosarcoma following combined chemotherapy with Adriamycin, vincristine, cyclophosphamide, and imidazole carboxamide. This patient remained disease-free 55 months after surgery. Shiu and coworkers $^{8}$ observed partial regression with radiotherapy, while Appleman and Helwig ${ }^{6}$ thought that radiation therapy has no beneficial effect on recurrence or survival. Graham and coworkers ${ }^{9}$ have advocated surgical resection as the major form of treatment, with no proven benefit from radiotherapy and chemotherapy.

\section{Summary}

In the case reported, a 47-year-old black woman presented with signs and symptoms of severe iron deficiency anemia. After diagnostic work up confirmed an intramural gastric mass with ulceration, abdominal exploration was performed. Intraoperative findings revealed a large, exogastric tumor identified as leiomyosarcoma; there was no evidence of gross metastasis. Radical subtotal gastrectomy with antecolic gastrojejunostomy was performed, and the patient had an unremarkable postoperative course. The patient remained disease free 20 months after gastric surgery.

Gastric leiomyosarcoma, a rare neoplasm of smooth muscle origin, presents with symptoms of weakness, gastrointestinal bleeding, pain, and weight loss. Diagnostic modalities include upper gastrointestinal radiography and selective celiac arteriography, endoscopy, and computerized to- mography. However, the superficial nature of the endoscopic biopsy forceps is not suitable to make a diagnosis of gastric leiomyosarcoma because of its intramural location and its frequent exogastric component. In our case, computerized tomography may have delineated the large exogastric component of the tumor.

The insidious onset of symptoms appears to be the reason why so many of these lesions grow quite large before they become clinically apparent. The majority of patients with gastric leiomyosarcoma develop metastasis, with the liver being the most commonly involved organ. Less-differentiated tumors and those with high pleomorphic activity have been found to metastasize earlier.

Surgical removal remains the treatment of choice. The neoplasm is completely resected with at least $2 \mathrm{~cm}$. of free margin allowed. The prediction of a 5-year survival rate is difficult for this as well as for any uncommon disease. The overall 5-year survival rate in the M.D. Anderson Tumor Institute and Hospital series ${ }^{4}$ was 19 percent. Further clinical trials must be carried out before the value of adjuvant chemotherapy and radiation therapy for gastric leiomyosarcoma can be assessed.

1. Chaudhuri, P.K., Nyhus, L.M., and DasGupta, T.: Sarcoma of the stomach. In Maingot's Abdominal operations, edited by S. Schwartz and H Ellis. Ed. 8. Appleton-Century-Crofts, East Norwalk, Connecticut, 1985 , pp. $995-1000$

2. Lindsay, P.C., Ordonez, N., and Raaf, J.H.: Gastric leiomyosarcoma Clinical and pathological review of fifty patients. Surg Oncol 18:399-421, 1981

3. Berk, R.N.: Sarcoma of the stomach. In Surgical radiology, edited by J.G. Teplick and M.E. Haskin. W.B. Saunders, Philadelphia, 1981, pp. 451-61

4. Scatarige, J.C., et al.: Gastric leiomyosarcoma. CT observations. J Comput Assist Tomog 9:320-7, Mar-Apr 85

5. Ranchod, M., and Kempson, R.L.: Smooth muscle tumors of the gastrointestinal tract and retroperitoneum. A pathologic analysis of 100 cases. Cancer 39:255-62, Jan 77

6. Appleman, H.D., and Helwig, E.B.: Gastric epithelioid leiomyoma and leiomyosarcoma (leiomyoblastoma). Cancer 38:708-28, Aug 76

7. Johnson, H., Hutter, J.J., and Paplanus, S.H.: Leiomyosarcoma of the stomach. Results of surgery and chemotherapy in an eleven-year-old girl with liver metastases. Med Pediatr Oncol 8:137-42, 1980

8. Shiu, M.H., et al.: Myosarcoma of the stomach. Natural history, prognostic factors and management. Cancer 49:177-87, Jan 82

9. Graham, S.M., Ballantyne, G.H., and Modlin, I.M.: Gastric epithelial leiomyosarcoma. A curable gastric neoplasm. Am J Gastroenterol 82:82-5, Jan 87

Accepted for publication in June 1986. Updating, as necessary, has been done by the authors.

Dr. McGarrigle is a resident in general surgery, Metropolitan Hospital, Springfield Division, and Dr. Gotfried is residency program director and chairman of the Department of Surgery.

Dr. McGarrigle, Metropolitan Hospital, Sproul Road and Thomson Avenue, 190 West Sproul Road, Springfield, Pennsylvania, 19064-2097. 


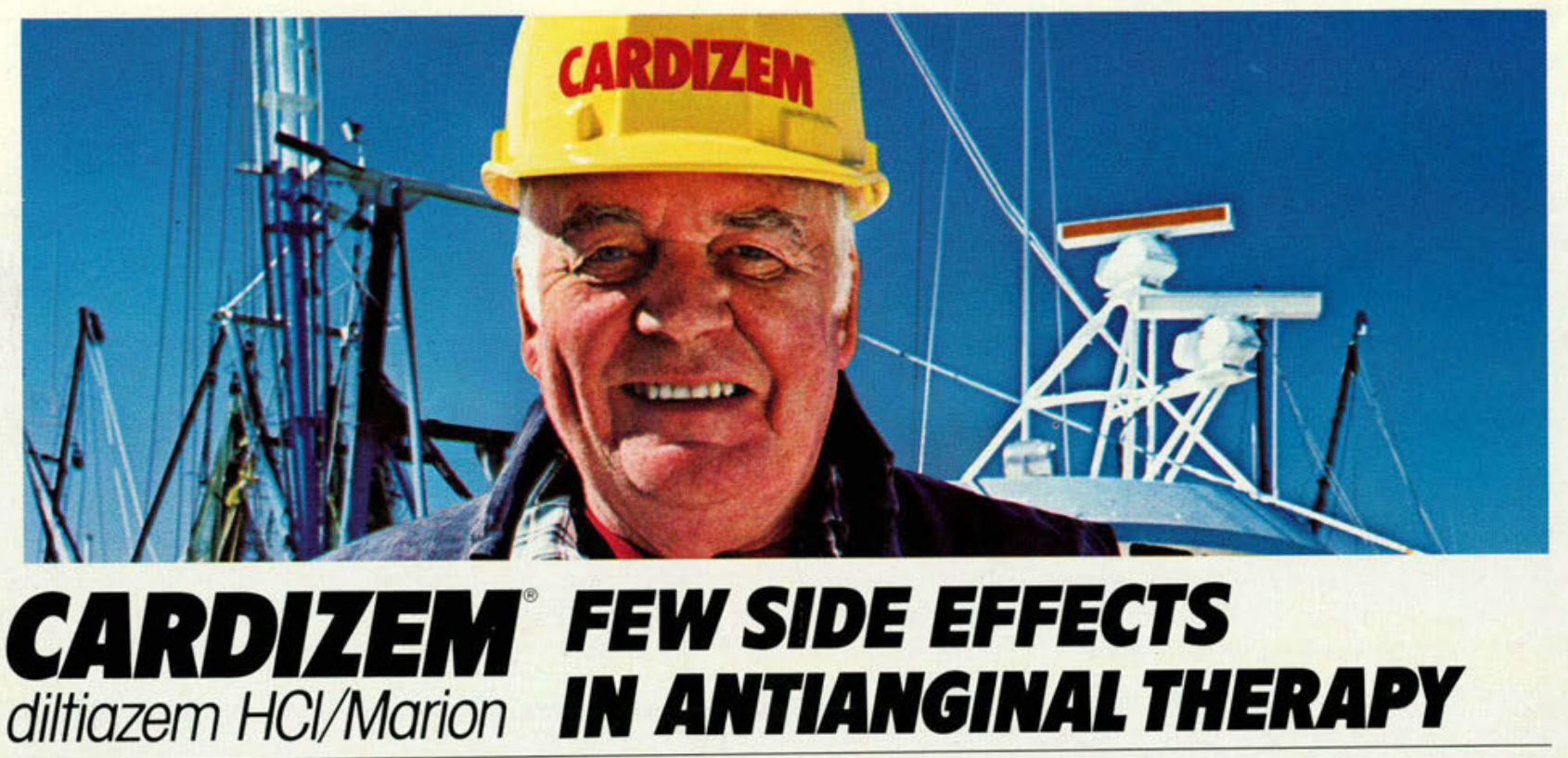

$60 \mathrm{mg}$ tid or qid

Brief Summary

Professional Use Information

\section{CARDIZEM}

(dilitiazem HCl) $30 \mathrm{mg}$ and $60 \mathrm{mg}$ Tablets

\section{CONTRAINDICATIONS}

CARDIZEM is contraindicated in (1) patients with sick sinus syndrome except in the presence of a functioning ventricular pacemaker (2) patients with second- or third-degree AV block except in the presence of a funcfioning ventricular pacemaker, and (3) patients with hypotension (less than $90 \mathrm{~mm} \mathrm{Hg}$ systolic).

\section{WARNINGS}

Cardiac Conduction. CARDIZEM prolongs AV node refractory periods without significantly prolonging sinus node recovery time, except in patients with sick sinus syndrome. This effect may rarely result in abnormally slow heart rates (particularly in patients with sick sinus syndrome) or second-or third-degree AV block (six of 1,243 patients for $0.48 \%$ ) Concomitant use of diltiazem with beta-blockers or digitalis may result in additive effects on cardiac conduction. A patient with Prinzmetal's angina developed periods of asystole (2 to 5 seconds) affer a single dose of $60 \mathrm{mg}$ of dilfiazem.

2. Congestive Heart Failure. Although dilfiazem has a negative inotropic effect in isolated animal tissue preparations, hemodynamic studies in humans with normal ventricular function have not shown a reduction in cardiac index nor consistent negative effects on contractility ( $d p / d t)$.

Experience with the use of CARDIZEM

alone or in combination with beta-blockers in patients with impaired ventricular function is very limited. Caution should be exercised when using the drug in such patients.

3. Hypotension Decreases in blood pressure associated with CARDIZEM therapy may occasionally result in symptomatic hypotension.

4. Acute Hepatic Injury. In rare instances, significant elevations in enzymes such as alkaline phosphatose, CPK, LDH, SGOT, SGPT, and other symptoms consistent with acute hepotic injury have been noted. These reactions have iveen reversible upon discontinuation of drug therapy. The relationship to CARDIZEM is uncertain in most cases, but prob able in some. (See PRECAUTIONS.)

\section{PRECAUTIONS}

General. CARDIZEM (diltiozem hydrochloride) is extensively metabolized by the liver and excreted by the kidneys and in bile. As with any new drug given over prolonged periods, laboratory parameters should be monitored at regular intervals. The drug should be used with caution in patients with impaired renal or hepatic function. In subacute and chronic dog and rat studies designed to produce toxicity, high doses of diltiazem were associated with hepatic damage. In special subacute hepatic studies, oral doses of $125 \mathrm{mg} / \mathrm{kg}$ and higher in rats were associated with histological changes in the liver which were reversible when the drug was discontinued. In dogs, doses of $20 \mathrm{mg} / \mathrm{kg}$ were also associated with hepatic changes; however, these changes were reversible with continued dosing.

Drug Interaction. Pharmacologic studies indicate that there may be additive effects in prolonging AV conduction when using beta-blockers or digitalis concomitantly with CARDIZEM. (SEe WARNINGS.)

Controlled and uncontrolled domestic studies suggest that concomitant use of CARDIZEM and beta-blockers or digitalis is usually well tolerated. Available data are not sufficient, however, to predict the effects of concomitant treatment, particularly in potients with left ventricular dysfunction or cardiac conduction abnormalities. In healthy volunteers, dilfiazem has been shown to increase serum digoxin levels up to $20 \%$.

Corcinogenesis, Mutagenesis, Impaiment of Fertility. A 24-month study in rats and a 21-month study in mice showed no evidence of carcinogenicity. There was also no mutagenic response in in vitro bocterial tests. No intrinsic effect on fertility was observed in rats. Pregnancy. Category C. Reproduction studies have been conducted in mice, rats, and rabbits. Administration of doses ranging from five to ten fimes greater (on a mg/kg basis) than the daily recommended therapeutic dose has resulted in embryo and fetal lethality. These doses, in some studies, have been reported to cause skeletal abnormalities. In the perinatal postnatal studies, there was some reduction in early individual pup weights and survival rates. There was an increased incidence of stillbitths ot doses of 20 times the human dose or greater.

There are no well-controlled studies in pregnant women; therefore, use CARDIZEM in pregnant women only if the potential benefit justifies the potential risk to the fetus.

Nursing Mothers. Diltiazem is excreted in human milk. One report suggests that concentrations in breast milk may approximate serum levels. If use of CARDIZEM is deemed essential, an alternative method of infant feeding should be instituted.

Pediatric Use. Safety and effectiveness in children have not been established.

\section{ADVERSE REACTIONS}

Serious adverse reactions have been rare in studies carried out to date, but it should be recognized that patients with impaired ventricular function and cardioc conduction abnormalities have usually been excluded.

In domestic placebo-controlled trials, the incidence of adverse reactions reported during CARDIZEM therapy was not greater than that reported during placebo therapy

The following represent occurrences observed in clinical studies which can be at least reasonably asso- ciated with the pharmocology of calcium influx inhibition In many cases, the relationship to CARDIZEM has not been established. The most common occurrences as well as their frequency of presentation are: edema (2.4\%), headoche $(2.1 \%)$, nousea $(1.9 \%)$, dizziness $(1.5 \%)$, rash $(1.3 \%)$, asthenia (1.2\%). In addition, the following events were reported infrequently (less than $1 \%$ )

Cardiovoscular: Angina, arrhythmia, AV block (first degree), AV block (second or third degree - see conduction warning) brodycardia, congestive heart failure, flushing, hypotension, palpitotions, syncope.

Nervous System: Amnesia, gait abnormality, hallucinations, insomnia, nervousness, paresthesia, personality change, somnolence, tinnitus, tremor.

Gastrointestinal: Anorexia, constipation, diamthed dysgeusia, dyspepsia, mild elevations of alkaline phosphatase, SGOT, SGPT, and LDH (see hepatic warnings), vomiting, weight increase.

Dermatologic: Petechioe, pruritus, photosensitivity, unticaria.

Other:

Amblyopia, dyspnea, epistaxis, eye imitation, hyperglycemia, nasal congestion, nocturia, osteoarticular pain, polyuria, sexual difficulties.

The following postmarketing events have been reported infrequently in patients receiving CARDIZEM alopecia, gingival hyperplosia, erythema multiforme, and leukopenia. However, a definitive cause and effect between these events and CARDIZEM therapy is yet to be established.

See complete Professional Use Information before prescribing.

References: 1. Pepine CJ, Feldman RL, Hill JA et a Clinical outcome after treatment of rest angina with calcium blockers: Comparative experience during the initial year of therapy with diltiazem, nifedipine, and verapamil. Am Heart J 1983; 106(6): 1341-1347. 2. Shapiro W: Calcium channel blockers: Actions on the heart and uses in ischemic heart disease. Consultant 1984:24(Dec): 150-159. 3. Johnston DL, Lesoway R Humen DP, et al: Clinical and hemodynamic evaluation of propranolol in combination with verapamil, nifedipine and diltiazem in exertional angina pectoris: A placebocontrolled double-blind, randomized, crossover study Am J Cardiol 1985:55:680-687 4. Cohn PF, Brounwald E: Chronic ischemic heart disease, in Brounwald E (ed): Heart Disease: A Textbook of Cardiovascular Medicine ed 2. Philadelphia, WB Saunders Co, 1984, chap 39. 5. Schroeder JS. Calcium and beta blockers in ischemic heart disease: When to use which. Mod Med

1982:50(Sept):94-116 